•综述・

\title{
无人机高光谱影像与冠层树种多样性监测
}

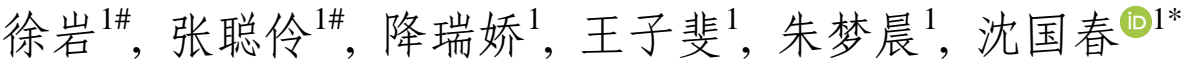

华东师范大学生态与环境科学学院, 浙江天童森林生态系统国家野外科学观测研究站, 上海 200241

摘要: 冠层树种多样性是自然森林生态系统功能和服务的重要基础。及时掌握冠层多样性的现状及变化趋势, 是探讨诸多重 要生态学问题的前提, 更是制定合理生物多样性保护策略的基础。但受制于传统的多样性信息采集方法，区域尺度的高精度 冠层多样性监测发展较为缓慢; 许多在气候变化和人类干扰下的生物多样性分布信息得不到及时更新。近年来基于无人机的 冠层高光谱影像收集与分析技术的发展, 使得冠层多样性监测迎来了新的发展契机。本文从森林冠层高光谱影像出发, 介绍 了与多样性监测相关的无人机航拍和基于深度学习的图像处理技术, 并结合已有文献, 探讨了无人机高光谱应用于森林冠层 树种多样性监测的研究现状、可行性、优势及缺陷等。我们认为冠层高光谱影像为多样性监测提供了不可或缺且丰富的原始 信息; 而无人机与高光谱相机的结合, 使得区域化高频率(如每周)、高精度(如分米乃至厘米级)的冠层多样性信息自动化收集 成为可能。然而高光谱影像数据量大、数据维度高与数据结构非线性的特点为影像处理带来了挑战, 而深度学习技术的飞跃, 使得从冠层高光谱影像中提取个体及物种信息达到了极高精度。恰当地使用这些技术将大大提升冠层树种多样性的自动化监 测水平, 由此也将帮助我们在当前剧变环境下及时掌握森林冠层多样性的现状与变化, 为生物多样性研究与保护提供可靠的 数据支撑。

关键词: 冠层多样性; 多样性监测; 高光谱影像; 无人机遥感; 深度学习

徐岩, 张聪伶, 降瑞娇, 王子斐, 朱梦晨, 沈国春 (2021) 无人机高光谱影像与冠层树种多样性监测. 生物多样性, 29, 647-660. doi: 10.17520/biods.2021013.

Xu Y, Zhang CL, Jiang RJ, Wang ZF, Zhu MC, Shen GC (2021) UAV-based hyperspectral images and monitoring of canopy tree diversity. Biodiversity Science, 29, 647-660. doi: 10.17520/biods.2021013.

\section{UAV-based hyperspectral images and monitoring of canopy tree diversity}

Yan $\mathrm{Xu}^{1 \#}$, Congling Zhang ${ }^{1 \#}$, Ruijiao Jiang ${ }^{1}$, Zifei Wang ${ }^{1}$, Mengchen Zhu ${ }^{1}$, Guochun Shen ${ }^{1 *}$

Zhejiang Tiantong Forest Ecosystem National Observation and Research Station, School of Ecological and Environmental Sciences, East China Normal University, Shanghai 200241

\begin{abstract} development of new tools for canopy diversity monitoring.

收稿日期: 2021-01-12; 接受日期: 2021-03-16

基金项目: 国家自然科学基金(31870404)

\# 共同第一作者 Co-first authors

* 通讯作者 Author for correspondence. E-mail: gcshen@des.ecnu.edu.cn
\end{abstract}

Background \& Aims: The species diversity of canopy trees is important for the function and service of natural forest ecosystems. To be able to formulate reasonable biodiversity conservation strategies, it is important to understand patterns of forest canopy diversity through time. However, the development of high-precision forest canopy diversity monitoring at a regional scale is slow due to a limitation in diversity information collection methods. A lot of biodiversity patterns may substantially change due to climate change and human disturbance. However, updating these changes in biodiversity cannot be done in a timely manner. In recent years, the development of canopy hyperspectral image collection based on unmanned aerial vehicle (UAV) and analysis technology has provided an opportunity for the

Progresses: Here, we propose using the hyperspectral image of forest canopy for biodiversity monitoring and conservation, development of UAV aerial photography and spatial positioning technology, and the development of hyperspectral image processing technology with deep learning. We use the existing literature to discuss the research status, feasibility, advantages, and disadvantages of using UAV hyperspectral imaging for monitoring of species diversity of forest canopy trees. We believe that canopy hyperspectral images provide indispensable and abundant 
information for forest biodiversity monitoring. The combination of UAVs and hyperspectral cameras makes it possible to automate the collection of canopy diversity information with both high frequency (e.g., weekly) and high precision (e.g., decimeter- or centimeter-level) at the regional scale. At the same time, the leap in image processing technology made possible through deep learning enables the extraction of individual and species information from canopy hyperspectral images with extremely high precision.

Prospects: Hyperspectral images have rich spectral and spatial information, which greatly improves the identification accuracy of plant species. The combination of UAVs and hyperspectral cameras greatly reduces the difficulty and cost for acquisition of this data. Applying deep learning methods to hyperspectral image processing can effectively collect species diversity information contained in hyperspectral images, and accelerate the research on forest canopy diversity monitoring on a large-scale. However, due to an insufficient sample size of hyperspectral data for species and a limitation in common deep learning models not being fully optimized for hyperspectral images remains a challenge. Future challenges for research include: how to build the hyperspectral species database, how to combine the characteristics of hyperspectral data, and how to optimize the automatic species recognition algorithm.

Key words: canopy diversity; diversity monitoring; hyperspectral image; UAV remote sensing; deep learning

在当前人类纪世代, 全球气候与土地利用方式 正经历剧烈的变化, 由此导致的物种分布和数量也 都在快速地改变。人类赖以生存的生态系统有 $60 \%$ 正处于退化或者不可持续利用状态(http://www.mill enniumassessment.org/zh/Condition.html)。据预测 , 在日益加剧的人类活动影响下, 现在的生物物种灭 绝速度至少是自然灭绝的1,000倍, 比形成速度快 100万倍(赵士洞和张永民, 2006)。在此背景下, 如何 更好地开展生物多样性调查、研究与保护是亟待解 决的重要生态学问题。20世纪中叶至今, 生物多样 性调查研究取得了长足的进步, 比如相关研究表明, 多类型的栖息地常常拥有丰富的物种 (Kerr \& Ostrovsky, 2003; Luoto et al, 2004); 物种多样性分 布与气候因子(温度、降水和能量等)和地形因子(高 程、坡度等)存在一定的关系(单峰或降低趋势) (Bradford et al, 2003; Duro et al, 2007)等。但由于时 间与空间尺度的限制, 许多基本的物种多样性保护 问题尚未得到很好的解答。例如, 在物种多样性快 速丧失的今天, 仍然缺乏一张准确且及时更新的物 种分布地图, 用于反映哪些物种的种群数量在急剧 减少、哪些物种的分布范围在快速变化。尽管中国 科学院和环境保护部已经联合编写了《中国生物多 样性红色名录》、《中国生物物种名录》等, 但数据 缺乏等级的物种仍达上千种, 这些物种缺乏研究和 野外调查工作, 人类对其生存现状了解甚少 (http://www.sp2000.org.cn/); 特别是对于那些生长 在森林深处的濒危植物而言, 它们的种群现状及动 态信息极度缺乏。这些关键信息的缺失给物种多样 性的保护实践带来极大的不确定性与风险。例如在
缺乏主要或关键物种分布信息的背景下，如何精确 合理地规划自然保护区界线与功能分区? 如何实 现社会发展与生态保护的共赢? 在气候变化背景 下，是否有必要调整已有保护区界线和功能划分?

同样，因为缺乏详细且及时更新的物种分布信 息, 生物多样性研究领域的许多重要生态学理论问 题也都悬而未决。例如, 受经典野外样方调查面积 的限制, 植物群落生态学研究的样方尺度很少突破 25 ha (Anderson-Teixeira et al, 2015), 因此很难实现 区域范围(特别是在人迹罕至的边远地区)的物种多 样性监测, 这就限制了生态学家对于种子扩散等具 有较大空间尺度的生态过程的详细研究与理解 (Wang \& Smith, 2002)。此外, 受野外人工调查的限 制, 生态学家较难开展时间和空间上较为密集的多 样性调查, 也很难获得区域范围的植物群落功能性 状等的空间格局特征(张志明等, 2017), 这在很大程 度上妨碍了研究者们对生境破碎化如何影响群落 动态和生物多样性的理解。在有限的人力与物力条 件下, 许多物种多样性调查样方仅覆盖了研究区域 面积的 $1 \%$ 以下; 而且由于许多调查数据更新周期 长, 难以真实反映当今剧烈环境变化下物种多样性 的现状。为克服这些难题, 已有一大批理论生态学 家致力于探索不同时空尺度下生物多样性格局的 尺度推绎(scaling)规律(Chesson et al, 2005)。然而由 于在不同时空尺度上占主导地位的格局和过程不 同、空间异质性因尺度而异等原因, 影响物种分布 的因素纷繁复杂, 尺度推绎理论进展缓慢; 并且精 确检验这些理论依然需要较大尺度的生物多样性 调查数据作为依据(邬建国, 2007)。 
在此背景下, 发展一种自动高效的多样性调查 方法对于生物多样性研究与保护具有重要理论与 实践意义。本研究聚焦于利用森林冠层影像开展林 冠层植物多样性监测的最新发展趋势。森林林冠层 植物不仅在地球生态系统的碳氮养分循环中扮演 着极其重要的角色, 而且是人类社会许多生产资料 (如木材)的提供者以及区域生态安全的重要屏障 (宋永昌, 2001)。研究与监测森林林冠层植物多样性 对了解整个森林生物多样性具有重要的意义。

但通过冠层影像开展冠层物种多样性监测并 非易事。第一, 绝大部分树种的林冠都呈现相似的 绿色(图1A), 使得基于普通RGB影像的物种识别极 其困难(谭炳香等, 2008)。这种限制在物种多样性较 高的群落中尤为突出。第二, 虽然不同物种的林冠 在不同物候期可能会呈现不同的色彩, 但受限于作 为冠层影像来源的卫星访问周期, 要获得丰富、连 续的不同物候期冠层影像数据成本较高, 而且这些 影像分辨率往往在米级别或以上(郭庆华等, 2016), 这使得基于冠层影像的物种识别更加困难。第三, 即使获取了高时空精度的冠层影像, 如何从中准确 提取植物物种和个体信息又是一道难题。

近些年各种技术的飞速发展使得解决上述三 个难点成为可能, 为基于冠层影像的植物多样性监 测提供了新方法。首先, 高光谱相机成本降低, 这
使得收集冠层高光谱影像不再是极其昂贵的步骤, 大量高光谱冠层影像为物种识别和个体定位提供 了原始数据; 其次, 无人机和空间定位技术的发展 使得生态学家也能轻松操控无人机进行冠层影像 拍摄, 这为大面积、高频率的冠层多样性监测提供 了数据收集的技术方案; 最后, 更强大的计算机硬 件和便于掌握的深度学习应用算法框架, 为图像分 析带来了革命性的改变, 使得从冠层影像中提取个 体及物种信息成为可能(Wäldchen \& Mäder, 2018)。

本文将从森林冠层高光谱影像出发, 介绍与多 样性监测相关的无人机航拍和基于深度学习的图 像处理技术; 并结合已有文献，探讨应用无人机高 光谱摄影对森林冠层树种多样性监测的研究现状、 可行性、优势等; 最后基于现有研究的难点和缺陷, 探讨该领域今后发展的重要研究方向。

\section{森林冠层高光谱影像}

典型的冠层高光谱影像与普通相机拍摄的 RGB影像没有本质区别。但普通RGB图片只有红绿 蓝3个颜色通道(图1A), 而高光谱影像有几百个颜 色通道, 其中每一个颜色通道代表某一波段光的相 对反射强度(图1B)。将图像中的某一个像素包含的 所有光谱信息组合起来, 就可以得到该像素点所在 林冠位置的近乎连续的光谱反射曲线(图1C)。
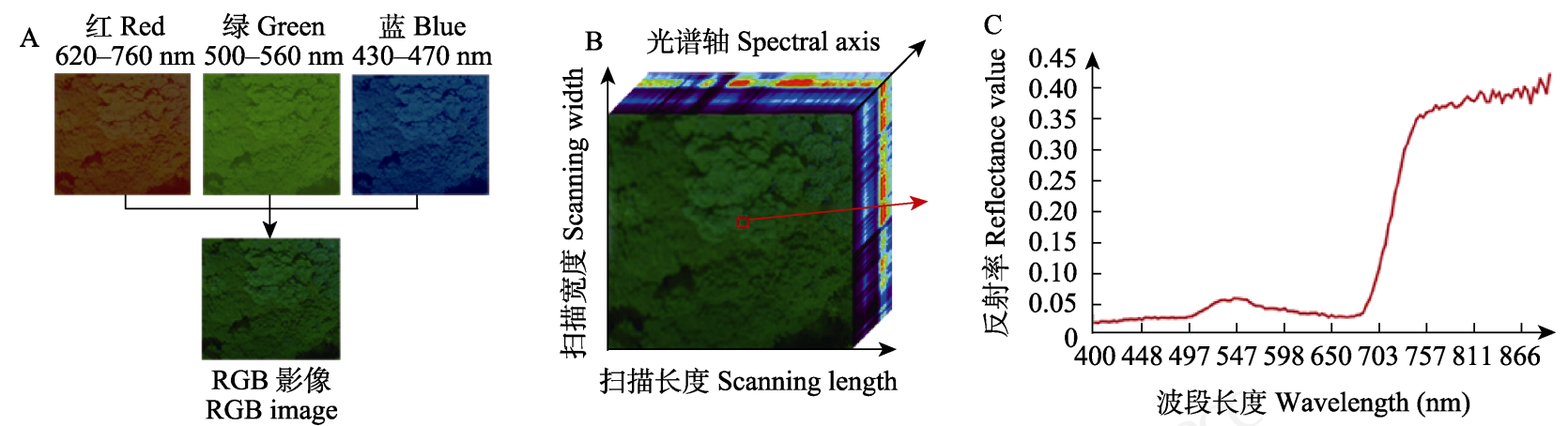

图1 浙江天童亚热带常绿阔叶林典型森林冠层RGB影像与高光谱影像示意图。(A)普通RGB影像, 仅包含红(620-760 nm)、 绿 $(500-560 \mathrm{~nm})$ 、蓝 $(430-470 \mathrm{~nm}) 3$ 层信息, 因此在RGB影像中多数常绿树种的冠层呈现近乎相同的绿色, 给冠层树种的识别 造成了极大的困难; (B)冠层高光谱影像的三维立体展示, $x$ 轴为扫描长度, $y$ 轴为扫描宽度, $\mathrm{z}$ 轴为光谱轴; (C)选定像素的光谱 反射曲线, 横坐标代表波长, 纵坐标代表波段反射率值, 该像素在不同的波段下表现出不同的反射率值, 组成了一条近乎连 续的光谱曲线。

Fig. 1 RGB image and hyperspectral image of typical forest canopy of the subtropical evergreen broad-leaved forest in Tiantong, Zhejiang Province. (A) Ordinary RGB images only contain three layers of information: red (620-760 nm), green (500-560 nm) and blue (430-470 nm). Therefore, the canopy of most evergreen tree species is almost the same green in RGB images, which makes it very difficult to identify the canopy species; (B) Three dimensional display of canopy hyperspectral image, $x$-axis is the scanning length, $y$-axis is the scanning width, z-axis is the spectral axis; (C) The spectral reflection curve of selected pixel, abscissa represents the wavelength, ordinate represents the band reflectance value, the pixel shows different reflectance values in different bands, forming a nearly continuous spectral curve. 
以图2的冠层高光谱影像为例, 在普通RGB冠 层影像上几乎无法区分不同物种的冠层信息。通过 主成分分析(principal component analysis, PCA)提取 冠层高光谱影像的主要信息后, 就能看到不同树种 的林冠呈现出不同的颜色(图2)。这就意味着在普通 RGB影像上都是绿色的植物, 在不同的光谱曲线上 存在可区别的颜色差异, 说明高光谱影像具备充分 潜力, 能够反映出不同树种之间的细微差异。

不同物种和不同树木个体间存在可测量的光 谱差异, 是基于冠层高光谱影像的物种多样性监测 的基础。具体而言, 当太阳辐射到达植物时, 可能 被反射、吸收或转化。所有植物都含有能形成反射 光谱的基本成分, 包括叶片色素、水分、蛋白质、 淀粉、蜡和其他结构分子, 如木质素和纤维素。常 用于物种分类的高光谱波段范围为 $400-2,500 \mathrm{~nm}$, 其中包括可见光部分(VIS, 400-700 nm)、近红外部 分 (NIR, 700-1,000 nm) 和短波红外部分 (SWIR, 1,000-2,500 nm) (Adam et al, 2010)。在绿叶中, 光合 色素(叶绿素 $a$ 和叶绿素 $b$ ) 和辅助色素在可见光波段 范围内表现出较高的吸收率; 水分则在近红外较窄 波段表现出吸收特征(Rivard et al, 2008); 在短红外 波段内, 具有高信噪比的高光谱传感器可以探测到 木质素、纤维素、蛋白质等吸收特征信息(Clark \& Roberts, 2012)。除化学组成外, 影响光谱的因素还 包括植物组织内部和表面的结构, 这些会影响光子 的散射和传输。植物的独特生化和结构特性可以转 化为物种水平的光谱特性, 而高光谱传感器可以探 测到这些光谱特异性(Adam et al, 2010)。目前已有 越来越多的证据表明, 不同植物物种所具有的独特 光谱特征能被高光谱传感器所探测 (Asner et al, 2009)。图3展示了 4 个不同物种(包含5个不同个体) 的光谱特征曲线, 从中可以清晰地看到, 这些物种 的光谱具有明显差异, 同一物种不同个体间也存在 光谱差异, 这虽然向基于高光谱的物种分类提出了 挑战, 但也为种间与种内的物种多样性监测提供了 丰富的数据基础。

\section{2 无人机航拍与空间定位}

生物多样性监测不仅需要收集物种的光谱信 息, 还需要知道具体有哪些物种, 并了解这些物种 的时空分布。这些时空信息需要自动高效的数据收 集手段作为支撑。近些年来, 无人机近地面遥感技
术不断发展完善(无人机近地面遥感系统工作示意 图见附录1), 具有系统性、即时性、非破坏性的遥 感技术可实现长期标准化地重复对地观测, 为多样 性监测提供了及时并且相对准确的信息(Adam et al, 2010; 唐志尧等, 2018)。相较于传统的实地样方调 查, 利用无人机成像平台进行冠层植物多样性监测 可以节省大量的人力物力; 与传统卫星与有人机遥 感手段相比, 无人机近地面遥感平台具有高时效/ 高机动性、高时空分辨率和低费用等优势(胡健波和 张健, 2018), 能够获得更高质量的野外观测数据。因 此, 无人机遥感是一种高性价比的工具, 使得生态 学研究者能够以鸟瞰的视角获取森林冠层的数据。

随着我国北斗导航网络的建成和千寻位置服 务的普及, 无人机的空间定位能力有了极大的提 升。在手机移动网络信号较好的区域, 无人机可以 实现厘米级的实时空间定位。我们不仅可以使用无 人机定期拍摄冠层影像, 而且可以拍摄同一位置同 一角度的不同时期影像, 从而大大简化了后期冠层 多样性监测影像的比较分析流程。在无手机信号的 偏远山区, 同样可以通过后差分技术获得厘米级的 空间位置信息。此外, 目前无人机飞控系统已经能 够实现全程自主飞行(兼容手动飞行)、多元化空间 数据获取、精准地形变高飞行等功能。国内无人机 技术已处于世界顶尖行列, 已有平台开发出多种机 型的无人机, 能搭载如激光雷达、一般可见光、多 光谱、高光谱在内的多款相机, 以及最近开发的无 人机自动起降平台, 能满足生态学家对于研究区域 多源、多时相的监测需求(https://enterprise.dji.com/ cn/surveying/natural-resource-management?site=enter prise\&from=nav); 某些具有测绘级别的无人机还可 实现对研究区域的精准定位, 显著提升了图像元数 据的绝对精度(http://www.feimarobotics.com/zhcn/in dustryAppDetail?newsID=11)。

然而, 利用无人机收集高光谱数据需要考虑光 照、风速等天气因素; 在实际飞行过程中, 为了覆 盖大片区域, 一般需要设定多条飞行路线, 这些路 线会受到采集时间、太阳角度、天气条件和潜在的 不同植物物候状态的影响(Fricker et al, 2019), 所以 在飞行之前需要进行一定量的前期准备工作, 如无 人机飞行航线预设、反射率设置, 飞行安全保障等, 以保证高光谱影像的质量。相对于RGB影像, 高光 谱影像具有丰富的光谱信息, 对太阳高度角等外界 

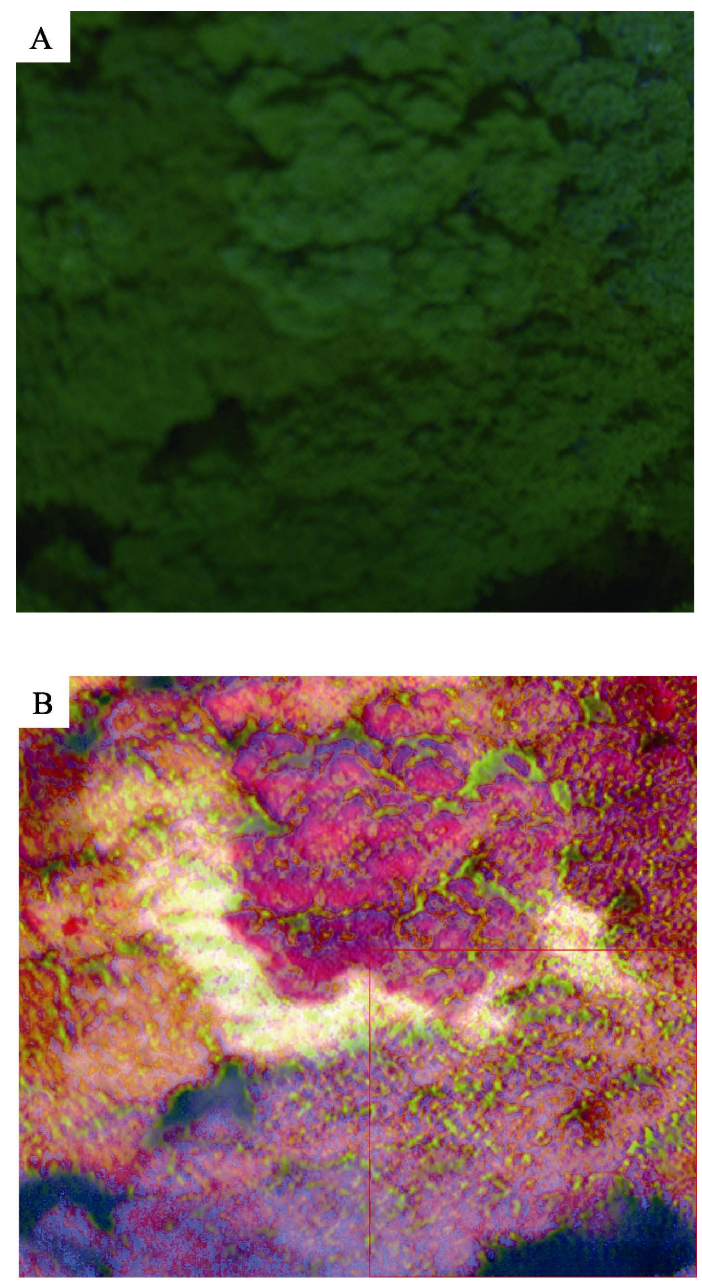

图2 冠层RGB影像与主成分分析(PCA)处理后的冠层高光 谱影像对比图。(A)冠层RGB影像, 各树种冠层呈现相近的 绿色; (B)通过PCA处理后的前三轴的冠层高光谱影像, 不 同树种的林冠呈现不一样的颜色, 这意味着高光谱影像具 备充分的潜力, 能够反映出不同树种之间的细微差异。

Fig. 2 Comparison of canopy RGB image and canopy hyperspectral image processed by principal component analysis (PCA). (A) The canopy RGB image shows that the canopy of each tree species is similar in green; (B) Through PCA processing of the first three axes of the canopy hyperspectral image, the canopy hyperspectral image of different tree species shows different colors, which means that the hyperspectral images have full potential to reflect the subtle differences between different tree species.

环境因素很敏感, 所以在飞行过程中也需要不断采 集白帧与暗背景以便于后续校准。对于不同类型的 高光谱相机收集到的数据, 处理方法有所不同。对 于内置推扫式高光谱相机, 图像拍摄结束后, 相片 需要依次进行镜头校正、反射率校正、大气校正等。 镜头校正主要用以纠正高光谱镜头成像的畸变; 反 射率校正用来校正在当前光照条件下物体的真实 反射率; 大气校正用于消除水汽等大气因素对于物

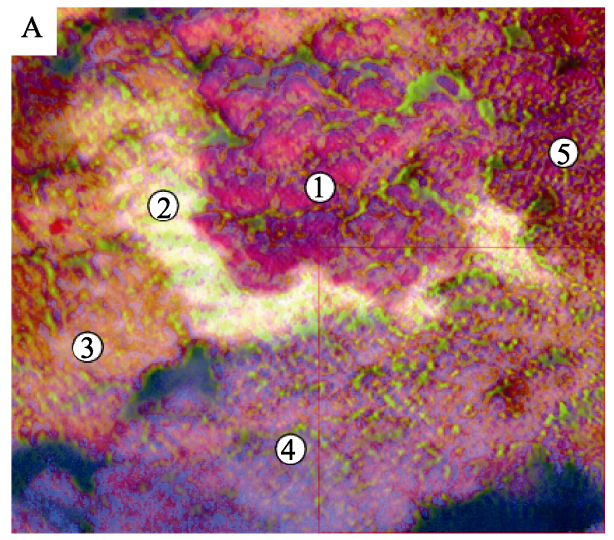

B 香樟1 Cinnamomum camphora 1 檫木 Sassafras tzumu 木荷 Schima superba 香樟2 Cinnamomum camphora 2

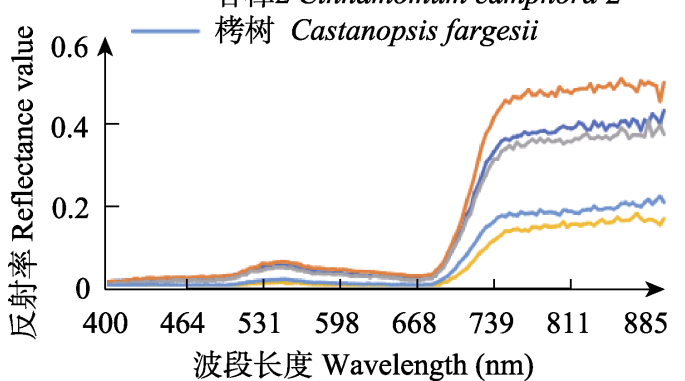

图3 个体的林冠层光谱特征曲线。(A)主成分分析(PCA)处 理后的冠层高光谱影像, 其中数字(1)-(5)分别代表不同的个 体。(B) 5 个林冠个体的光谱反射曲线。不同的植物因其化学 性质和结构的不同，表现出不同的光谱反射曲线，这是基于 光谱物种分类的基础。

Fig. 3 Individual canopy spectral characteristic curve. (A) In the hyperspectral images of forest canopy processed by principal component analysis (PCA), the numbers (1)-(5) represent different individuals; (B) Spectral reflectance curves of five canopy individuals. Different plants show different spectral reflectance curves because of their different chemical properties and structures, which is the basis of spectral species classification.

体反射的影响 ${ }^{1}$ 。相片校正结束后，需要将数据文件 集导入专业的拼接软件进行图像拼接。一般的拼接 软件利用经纬度信息及重投影(reprojection)算法细 化高光谱相机参数, 从而实现影像拼接(黄宇等, 2019)。但这种处理方式忽略了高光谱内丰富的光谱 信息。如果定位不够精准, 就会产生偏差; 当然也 存在一些商用软件可以利用高光谱的光谱特性辅 助拼接, 但目前仍处于不断发展过程中, 软件的功 能与稳定性逐步提升。对于画幅式高光谱相机，所

(1) 陆嘉辉 (2020) 基于林冠高光谱影像的树种分类研究. 硕士学位论 文, 华东师范大学, 上海. 
得的高光谱影像不需要经过镜头校正, 一般会在采 集高光谱影像的同时采集分辨率更高的灰度图像, 先将两者融合, 再与校正后的灰度图像进行影像配 准, 得到校正融合影像, 通过影像镶嵌、拼接、感 兴趣区域提取、平滑等步骤得到最终影像(秦占飞等, 2016)。而对于随飞行外置推扫式高光谱相机而言, 除了之前的镜头校正、反射率校正和大气校正外, 还需要利用二项反射分布函数 (bidirectional reflection distribution function, BRDF)进行正射归一 化校正, 采集的图像并不需要拼接, 而是直接利用 航带进行分析(荚文等, 2017)。当前无人机图像以及 多源数据处理技术日益强大, 一站式行业软件(常 用的一站式行业航测图像分析软件见附录2)的进步, 使得后期图像拼接、数据处理有了基本保障, 从而 使生态学家们能将更多精力投入到数据分析层面。

高光谱影像因无人机的优势在时间和空间分 辨率上得到了很大的提升; 高精度定位技术的发展 使得高光谱影像精度有了很大改善。无人机航拍与 空间定位技术的快速发展, 使高光谱相机有了更广 大的应用平台。在完成初始航线规划和首次监测后, 后期的定期定点冠层多样性监测仅需要将搭载高 光谱相机的无人机放在室外, 其他过程都可以通过 一站式软件自动化完成。因此, 高光谱相机与无人 机的结合, 使森林冠层多样性监测的时空尺度进入 一个全新的阶段 ${ }^{(1)}$ 。

\section{基于冠层影像的多样性信息提取}

高光谱影像在具有丰富光谱信息与空间信息 的同时, 也面临着数据量大、数据维度高与数据结 构非线性的挑战。高光谱影像的每个像素都包含了 数以百计的连续窄带光谱信息, 相邻波段的光谱高 度相关，因而收集到的高光谱往往包含大量冗余信 息，这极大地增加了数据处理的复杂性。不仅如此, 高光谱影像的大量波段数目会大幅度增加高光谱 数据的维度, 导致用于参数训练所需的样本数也急 剧增加。而在实际数据收集过程中, 样本量往往无 法满足需求, 过少的样本量无法保证参数的精度, 使得分类结果不理想。在成像过程中, 太阳发出的 电磁波经过折射、散射、吸收等作用后, 部分到达 目标区域并和周围地物进行交互作用，被反射后的

(1) 涂华听 (2017) 基于近地高光谱遥感数据的茶树分类和生化参数反 演. 硕士学位论文, 武汉大学, 武汉.
部分电磁波再次经过大气到达传感器，这些复杂的 成像过程也导致了高光谱数据的复杂性 ${ }^{2}$ 。这意味 着高光谱影像面临着数据量急剧增加、计算量增 大、信息冗余和非线性程度高等挑战，这就对数据 处理方法提出了更高的要求。

近年来我们共同目睹了深度学习给植物分类 带来的巨大进步与便利。人们可以通过手机app (如 花伴侣、形色等)拍照识别身边的花草植物。这些app 的后台都有强大的深度学习模型作为支撑。冠层物 种多样性监测也可以采用深度学习模型从冠层高 光谱影像中提取物种信息。深度学习模型类似于我 们熟悉的简单线性回归模型。这两类模型都需要给 定初始的模型结构, 其中包含待求解的未知参数; 这些未知参数需要通过自变量和因变量相关的观 测数据来估计; 参数估计方法也都是基于目标函数 的梯度下降法。但它们存在几点重要的区别：第一, 线性回归模型结构简单, 参数少 $(\leq 30$ 个), 仅能建 立自变量和因变量间的线性关系(Crawley, 2012); 第二, 深度学习模型结构复杂, 往往包含多个隐藏 层和上千万个参数; 第三，模型中包含许多非线性 单元，能建立变量间复杂的非线性关系(Ghamisi et al, 2018)。复杂的结构、海量的参数和非线性单元 的联合赋予深度学习模型极强的表达力，因此它特 别适合描述人类感觉上简单但又无法通过线性函 数关系描述的变量联系。例如, 植物物种间存在许 多差异, 这些差异可以通过人眼和深度学习模型辨 认，但它们很难通过人工定义的函数来表示。

深度学习模型中还有专门模拟生物处理图像 信息的网络结构，例如卷积神经网络模型 (convolutional neural network, CNN), 它非常适合从 影像中提取重要的特征信息，相关实例可见下文 5.2 节。卷积神经网络通过多层网络架构提取特征, 旨在模拟从视网膜到大脑皮层的传导过程，这些深 层结构在图像分类和目标检测方面有巨大潜力 (Chen et al, 2016)。如图4所示，一个完整的卷积神 经网络主要包括输入层、隐藏层和输出层。其中输 入层一般用于接收固定大小的影像; 输出层一般是 分类目标的概率输出; 中间的隐藏层包含卷积层、 池化层和全连接层。其中卷积层主要用于表示图像 中各种复杂的特征; 池化层一般紧随卷积层, 主要

(2) 林洲汉 (2014) 基于自动编码机的高光谱图像特征提取及分类方法 研究. 硕士学位论文, 哈尔滨工业大学, 哈尔滨. 


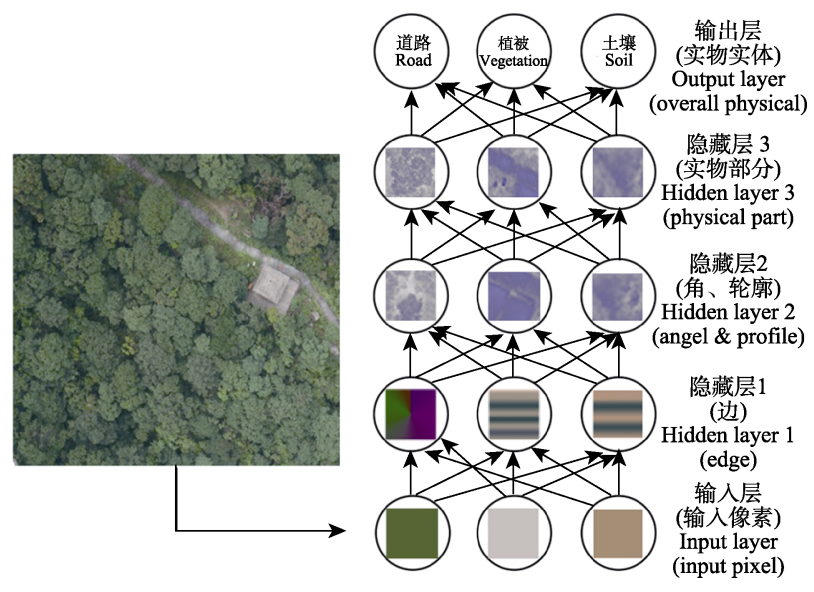

图4 基于深度学习网络的分类模型。模型中包括输入层、 隐藏层和输出层, 隐藏层用于提取图像特征, 层数越高, 隐 藏层提取的特征越高级。

Fig. 4 Classification model based on deep learning network. The model includes input layer, hidden layer and output layer. Hidden layer is used to extract image features. The higher the number of layers is, the higher the features can be extracted by hidden layer.

用于从各种复杂特征中提取主要特征; 全连接层则 当作分类器, 将传递到网络后端的重要特征与分类 目标紧密联系起来。具体的有关深度学习模型及在 生态学中的应用的相关介绍可参考Wäldchen和 Mäder (2018)、Christin等(2019, 2021)等。

\section{研究现状}

我们在 Web of Science 中分别输入 “hyperspect*” “deep learning” “UAV”或 “unmanned aerial vehicle”，并限定搜索类别为“ecology”，分别对 2000 年以来生态学研究领域使用高光谱、深度学习以及 无人机的文献进行检索, 得到的结果如图5所示。从 图中我们可以看出, 近些年来该领域的文献数量整 体呈上升趋势, 特别是 2015 年及以后发表的文献数 量大幅增加, 说明高光谱、无人机以及深度学习的 研究越来越受到生态学家的重视。下面我们将从不 同研究尺度分别对高光谱、无人机以及深度学习应 用于生物多样性的研究现状展开介绍。

\section{1 基于冠层光谱多样性的物种多样性调查}

由于生化特性与结构不同, 林冠层不同物种间 存在可测量的光谱差异, 所以光谱的多样性在某种 程度上可以直接反映物种多样性。有研究证明, 利 用高光谱特定波段计算出的植被指数与植物多样 性之间存在显著相关性(Fairbanks \& McGwire,

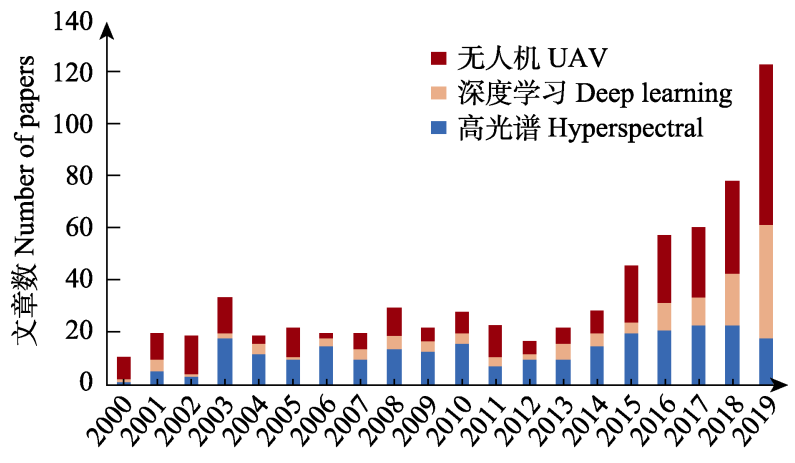

出版年份 Year of publication

图5 2000-2019年间生态学领域中分别使用无人机(UAV)、 深度学习、高光谱进行研究的文章统计结果

Fig. 5 From 2000 to 2019, the statistical results of articles in the field of ecology using unmanned aerial vehicle (UAV), deep learning and hyperspectral, respectively

2004; Cayuela et al，2006), 如归一化植被指数 (normalized difference vegetation index, NDVI)、增强 植被指数(enhanced vegetation index, EVI)等, 可以 解释区域内物种丰富度或多样性的变化。高光谱以 上百个连续且细分的光谱波段对目标区域同时成 像, 光谱分辨率非常高(Nagendra, 2001), 这就为大 尺度生物多样性监测提供了更为直接、简便的方式 (Oindo \& Skidmore, 2002; Turner et al, 2003)。

无人机和卫星是目前大面积高光谱数据采集 最主要的两个平台。近年来, 有许多研究应用星载 或机载高光谱进行区域尺度的物种多样性的研究, 并已取得重要进展。Kalacska等(2007)根据不同植物 叶片的光谱特征不同, 并结合物候变化, 利用高光 谱卫星图像研究了热带森林的结构与植物区系多 样性; 根据不同树种的光谱异质性，Kayet等(2020) 利用星载高光谱遥感对Sarandë地区的矿区森林进 行了基于Shannon多样性指数的多样性评估, 实现 了对矿区森林的有效监测与管理。与卫星遥感相比, 无人机作为近地面遥感平台, 具有小型化、数据收 集周期短以及空间分辨率高等优势，能获得更高质 量的高光谱影像。随着无人机机载技术的不断发展, 越来越多的生态学家更倾向于利用无人机来进行 生物多样性研究。例如在生物多样性监测和物种分 类领域, Vaglio Laurin等(2013)利用不同植物间的光 谱异质性，通过机载高光谱测绘了塞拉利昂国家公 园内林区冠层树种的 $\alpha$ 多样性; 董文雪 ${ }^{\circledR}$ 基于不同植

(1) 董文雪 (2018) 基于机载激光雷达及高光谱数据的亚热带森林乔木 物种多样性遥感监测研究. 硕士学位论文, 中国科学院遥感与数字地球 研究所, 北京. 
物间的结构与光谱特征的差异, 利用高光谱数据提 取䇥选植被指数, 在神农架国家级自然保护区开展 了亚热带森林乔木物种多样性监测, 实现了森林乔 木物种多样性的区域成图。一些学者还通过无人机 高光谱遥感进行了多样性格局及其驱动力的研究, 如Bongalov等(2019)在马来西亚热带雨林中利用机 载高光谱数据成功估算了该区域的 $\beta$ 多样性指数, 证明了高光谱遥感可用于 $\beta$ 多样性测算。在群落构 建理论的研究中, 无人机高光谱遥感还能从多样性 的各个维度(如功能、谱系等)助力群落构建过程的 理解, 如Asner等(2015)结合机载高光谱和激光雷达 技术, 评估了安第斯山 79个样地的冠层叶片的生化 特征, 并将研究扩展到整个秘鲁的热带雨林中, 测 量了不同冠层树种的叶片氮、磷含量等7个功能属 性，获得了该区域的功能多样性指数, 并绘制了大 区域尺度的森林冠层功能特征图(Asner et al, 2016, 2017)。此外, 在生物多样性保护领域, 由于外来物 种的光谱曲线一般与本地物种有所差别, 高光谱遥 感可以准确地探测到外来入侵种, 并预测其分布区 以及对当地物种多样性的影响(Underwood et al, 2003)。Asner和Vitousek (2005)根据固氮植物叶片氮 含量较高这一特征, 利用机载可见/红外高光谱成 像仪AVIRIS监测了夏威夷火山国家公园中入侵种 火杨梅(Myrica faya) 的入侵现状。以上案例表明, 光 谱多样性是衡量植物生物多样性的一个新兴维度, 它整合了物种内部和物种之间的性状变异, 即使在 缺乏分类、功能、系统发育或丰度信息的条件下，也 可开展高时空分辨率的调查与监测。

\section{2 基于物种自动识别的物种多样性调查}

除了直接使用光谱多样性反映物种多样性, 也 有一部分生态学家尝试使用机器学习算法处理高 光谱数据, 得到更精准的物种分类信息, 以进一步 开展物种多样性定量化研究。与区域尺度的光谱多 样性研究相比, 鉴别出区域内每个物种的空间分布 信息对生态学研究的意义更大, 但这也对研究技术 提出了更高的要求。首先要解决的问题是如何获得 物种的类别信息。在过去的 10 年中, 各种机器学习 算法在树种分类的应用上取得了显著进展(Fricker et al, 2019), 常见的分类算法如支持向量机(support vector machine, SVM)、随机森林(random forest, RF) 等, 已应用于(亚)热带干湿森林(Shen \& Cao, 2017), 温带、寒带森林(Dalponte et al, 2014, 2019; Ballanti et al, 2016)，人工种植林、农业林(Ghosh et al, 2014; Dalponte et al, 2015; Graves et al, 2016)和城市森林 (Pu, 2009; Zhang \& Qiu, 2012)等生态系统中。在处 理高光谱数据时，上述这些传统的分类算法尽管取 得了一定的成果，但由于仅提取低级特征并且过分 依赖人工选取的特征等原因，分类精度还有待提高 (奚雪峰和周国栋, 2016)。

近年来, 随着计算机技术的不断成熟及机器学 习领域的不断发展, 深度学习可以有效弥补传统分 类算法的不足, 在高光谱影像处理领域中表现出 色。研究发现, 利用深度学习网络提取的判别特征 可以明显提高对高光谱影像的物种分类性能 (Signoroni et al, 2019)。Hu等(2015)提出了基于光谱 数据的深度学习模型, 将其应用于 3 个公开的数据 集上，分类表现均优于传统的方法，最终分类准确 率超过了90\%; Xi等(2019)在中国长白山对7个树种 进行了基于高光谱影像的物种分类，结果表明利用 卷积神经网络搭建的分类模型精度(85.04\%)要显著 大于利用随机森林搭建的树种分类模型(80.16\%)。 以上案例说明深度学习是进行高光谱树种分类的 有效方法。为了更直观地展示深度学习在物种分类 领域的优势，我们收集了近15年来利用高光谱遥感 对植物进行物种识别的 66 个案例。使用Wilcoxon符 号秩检验对图6所示 4 组数据进行统计检验, 得到 $P$ $=0.01429$ 。结果显示，在不同的待分类物种数量下, 深度学习的分类精度均显著高于非深度学习(图6)。

$\square$ 深度学习 Deep learning 非深度学习 Non deep learning

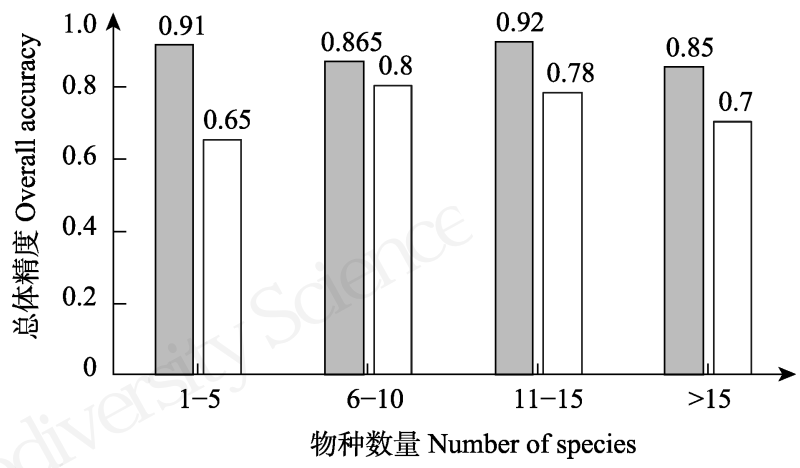

图6 深度学习与非深度学习算法在高光谱树种分类中的表 现。通过统计检验结果可看出, 深度学习算法在物种分类中 有明显优势。

Fig. 6 The performance of deep learning and non deep learning algorithms in hyperspectral tree species classification. The statistical test results show that the deep learning algorithm has obvious advantages in species classification. 
越来越多的研究表明, 随着深度学习技术的不 断完善与成熟, 基于深度学习的分类模型在不同森 林类型的树种分类任务中都有着良好的表现。在挪 威的亚寒带针叶林中, Trier等(2018)利用卷积神经 网络对云杉(Spruce)、松树(Pinus)、桦树(Birch) 3 个 树种进行了基于高光谱像素的分类, 准确度达到 87\%; 在北美西部的温带混合针叶林中, Fricker等 (2019)使用卷积神经网络和机载高光谱, 对白冷杉 (Abies concolor)、北美翠柏(Calocedrus decurrens)、 美国黑松(Pinus contorta) 在内的7个物种进行了识 别, 准确率达到 $87 \%$; 在波兰东南部的温带阔叶林 中, Raczko和Zagajewski (2018)在国家公园内对桤 木(Alnus cremastogyne)、落叶松(Larix gmelinii)等6 种温带阔叶林树种进行了基于高光谱和深度学习 的树种分类, 分类精度达到 $87 \%$; 在亚热带地区, 陆嘉辉 ${ }^{\circledR}$ 研究发现, 利用光谱和纹理信息的树种分 类模型精度优于仅用单一信息的模型, 并且将高光 谱应用于 162 个上海亚热带树种分类任务时, 深度 学习模型总体分类精度超过 $80 \%$ 。这些案例说明, 通过高光谱遥感和深度学习这两种新兴技术的结 合, 冠层树种的分类调查有望变得更加简便、高效, 这将极大地拓展生物多样性调查的广度与深度。

\section{发展方向}

高光谱影像具有丰富的光谱信息与空间信息, 使植物物种识别精度从原始数据上得到了很大程 度的提升; 无人机与高光谱相机结合大大降低了数 据获取的难度与成本; 将深度学习方法应用于高光 谱影像处理, 能够有效提取高光谱影像中包含的物 种多样性信息, 加速森林冠层多样性监测研究向自 动化、大范围的全新阶段迈进。但由于物种高光谱 数据样本量不足、常见的深度学习模型未针对高光 谱影像进行充分优化等问题, 该领域的发展受到了 限制。在之后的研究中, 如何构建高光谱物种数据 库, 如何结合高光谱数据的特征优化物种自动识别 算法, 是无人机高光谱冠层多样性监测所面临的主 要挑战。

\section{1 构建高光谱物种数据库}

深度学习为冠层植物分类提供了一套强大而 客观的工具, 具有巨大的潜力。但是深度学习模型

(1) 陆嘉辉 (2020) 基于林冠高光谱影像的树种分类研究. 硕士学位论 文, 华东师范大学, 上海.
需要大量的训练数据, 因此建立冠层物种光谱特征 库是当前基于冠层高光谱影像进行物种多样性监 测的迫切需求, 直接影响到无人机高光谱遥感在群 落及生态系统尺度中的应用。

然而, 目前公开的高光谱数据库较少, 且仅包 含少量的物种数, 这极大限制了深度学习在高光谱 影像上的应用。在研究中应用较多的是由机载可见 /红外成像光谱仪(AVIRIS)传感器在一片印第安农 业松林试验场收集得到的数据集Indian Pines, 它含 有695个训练集样本(像素)与9,671个测试集样本(像 素), 仅包含各类农作物与不同类型的耕地信息。另 一个常用的数据集是位于意大利的帕维亚城的 Pavia Centre and University, 该数据集通过反射光 学光谱成像系统机载仪器捕获, 由不同的类别组成, 包括树木、沥青、砾石、金属片、砖块、草地和土 壤。这个数据集包括3,921 个训练集样本(像素) 与 40,002个测试集样本(像素)。第三个应用较多的数据 集为grss_dfc_2013, 于2012年6月由小型航空光谱 成像仪 (compact airborne spectrographic imager, CASI)在美国德克萨斯州休斯顿大学校园及其邻近 城市地区捕获, 包括2,832 个训练集样本(像素)与 12,197 个测试集样本(像素), 空间分辨率为 $2.5 \mathrm{~m} 。$ 该数据集由0.38-1.05 nm范围的144个光谱波段组 成, 包括草地、树木、土壤、水、住宅、商业地区、 公路等15个类别(Ghamisi et al, 2018)。此外, 美国国 家生态观测站网络(United States National Ecological Observatory Network, NEON)还从2013年开始记录 光谱影像, 每年连续观测, 站点覆盖全美区域, 覆 盖范围广、持续时间长, 其中的高光谱分辨率为 $1 \mathrm{~m}$ (https://data.neonscience.org/data-products/explore.)。 但NEON站点只提供了原始的高光谱数据, 还未形 成一个大量的含完整物种标记信息的高光谱数据 集(少量标记的数据集可见https://zenodo.org/record/ 3934932\#.YDXiIegzY2x.)。

但是目前现有的高光谱数据集对森林冠层多 样性研究的适用性低。例如Indian Pines可用于植物 物种识别研究的样本量较少、空间分辨率较低且针 对性不强。而且不同的物种分类研究结果也因使用 不同高光谱数据等原因, 难以进行比较和归纳 (Thenkabail et al, 2018)。此外, 植被的生长发育具有 物候特性, 其光谱信息会随季节的变化而改变 (杨 超等, 2018)。因生长发育阶段、土壤、降水和地形 
等因素不同，同一个物种在不同的时空尺度下其光 谱也会发生变化。针对这个难题, 我们认为, 未来 可以借助公众科学(citizen science)的方式让大量科 学爱好者或志愿者共同参与物种信息标记, 随着信 息时代的到来和互联网技术的不断发展, 公众科学 在生态保护和监测等领域中的作用将越来越受到 重视(张健等, 2013), 借助公众科学的力量, 可以构 建大型公开且含有不同物候期植物物种的高光谱 数据集, 这将能极大促进森林冠层多样性的监测与 研究。

\section{2 针对高光谱影像特征构建合理的深度学习模型}

遥感技术在物种分类方面的快速发展得益于 模式识别、机器学习等领域的进步, 但现阶段该领 域仍处于快速发展阶段, 算法技术仍未成熟, 极大 地限制了物种分类精度的提高(孔嘉金等, 2019)。例 如, 如何充分挖掘高光谱遥感数据隐含的丰富信息, 是当前的一个技术难题(张扬建等, 2017); 如何设 计一个合适的卷积神经网络模型, 是机器学习和遥 感领域讨论的热门话题之一(Ghamisi et al, 2018)。 现有的卷积神经网络模型大多是一维或二维, 仅能 提取光谱特征或提取每个像素的局部空间特征, 导 致物种分类结果不佳(Chen et al, 2016)。三维卷积神 经网络可以利用空间光谱联合信息来处理高光谱 影像, 在一定程度上可以削弱同物异谱或者同谱异 物的影响，并且引入了空间背景信息、纹理、对象、 形状等特征和知识, 可以部分解决空间同质性与异 质性的问题，提高分类精度(杜培军等, 2016)。因此， 在此基础上进一步发展和完善三维卷积神经网络 模型十分必要。目前比较简单、可以广泛应用的深 度学习工具还比较少, 比如可视化的流程式深度学 习工具。所以, 寻找适合冠层高光谱影像的深度学 习网络结构是有待解决的主要问题之一。此外, 相 比于高精度的高光谱影像, 冠层反射率(canopy reflectance, CR) 数据较容易获得, 而许多冠层反射 率模型可以很好地描述植被的关键生化参数(如叶 面积指数、叶绿素含量、水分含量等)与冠层反射率 之间的物理机制(全兴文, 2017), 未来设想可以将冠 层反射率模型与深度学习相结合, 通过反射率模型 得到冠层光谱信息和植被关键生化参数, 进而进行 树种分类。

上述的模型只是对图像中的树种进行识别分 类, 但在实际的生物多样性监测与保护中, 还需要
对植物个体进行分割, 计算每个树种的个体数量。 因此在基于高光谱影像的树种分类中, 个体分割也 是十分重要的一个环节。为了使深度学习模型可以 实现这个目标, 首先需要我们在标记时以个体为单 位，并一一标明物种名称; 其次需要通过学科交叉 的方式来改进适合高分辨率高光谱影像的深度学 习模型, 如生态学家贡献出高光谱数据库或者物种 标记数据库, 以组织类似Kaggle竞赛的形式, 与专 业研究深度学习模型的图像分析人士合作, 开发出 更实用、更完善的物种分类模型。

\section{3 多元数据融合}

尽管高光谱数据有光谱分辨率高、能够识别森 林物种细小的波谱差异等优势，但相比于无人机普 通RGB影像，也存在着空间分辨率低、飞行条件要 求高、采集效率低等劣势。近些年来，随着物种分 类识别研究的不断深入, 复杂物种信息的提取需要 通过多元数据融合发挥出各类数据的优势。如融合 RGB影像可以得到更高的空间分辨率，更好地获取 物种冠层的纹理信息; 激光点云可获得林冠内部的 三维结构信息，通过结合三维信息也可用于区分不 同物种的林冠。例如, 基于传统光学数据, 光谱特 征差异明显的森林类型——针叶林和阔叶林分类 精度较高, 但是当森林物种类型较多时, 难以获得 理想的分类结果。而在结构复杂的森林生态系统中, 激光雷达由于穿透性好, 可以深入到冠层内部获取 数据, 提供森林冠层的三维结构信息。因此, 将高 光谱与激光雷达技术结合, 可获得植物的光谱特征 和结构特征，实现高精度的植物分类。所以通过数 据融合可以实现多源数据协同作业，解决在复杂生 境的结构参数提取、生物量估测以及植物物种识别 等领域的难题。但是在多源数据融合过程中存在数 据结构、精度等是否匹配的问题, 这些问题的解决 既需要更好的数据融合算法, 也需要从源头着手, 不断升级无人机硬件设备, 使无人机得以搭载多种 遥感仪器, 同时同地进行数据收集; 或进一步提升 每一种数据的空间定位精度，以方便不同数据的后 续结合。

综上所述，高光谱影像具有丰富的光谱信息与 空间信息，无人机航拍与空间定位技术的快速发展 使高光谱遥感越来越广泛地应用于生态学研究中。 将无人机高光谱遥感数据与能自动提取特征的卷 
积神经网络相结合，在森林冠层多样性研究领域有 着巨大的潜力。未来还需要在高光谱数据库、模型 结构、数据融合等方面进行进一步的研究, 以实现 大尺度、自动化、高精度的树种分类。这些技术在 降低野外监测的人力和物力成本的同时，可为复杂 的尺度推绎和大尺度生物多样性的估算提供数据 支持, 并为种群分布与动态监测、种间相互作用等 方面的研究提供真实可靠的观测数据, 最终为生物 多样性管理和保护等领域提供有力支撑。

致谢: 在本次研究过程中, 特别感谢华东师范大学 公共创新服务平台的仪器支持，十分感谢对这篇文 章提供宝贵意见的各位专家!

\section{ORCID}

沈国春 (D) https://orcid.org/0000-0001-9853-6062

\section{参考文献}

Adam E, Mutanga O, Rugege D (2010) Multispectral and hyperspectral remote sensing for identification and mapping of wetland vegetation: A review. Wetlands Ecology and Management, 18, 281-296.

Anderson-Teixeira KJ, Davies SJ, Bennett AC, Gonzalez-Akre EB, Muller-Landau HC, Wright SJ, Salim KA, Almeyda AM, Alfonso A, Baltzer JL, Basset Y, Bourg NA, Broadbent EN, Brockelman WY, Bunyavejchewin S, Burslem DFRP, Butt N, Cao M, Cardenas D, Chuyong GB, Clay K, Cordell S, Dattaraja HS, Deng XB, Detto M, Du XJ, Duque A, Erikson DL, Ewango CEN, Fischer GA, Fletcher C, Foster RB, Giardina CP, Gilbert GS, Gunatilleke N, Gunatilleke S, Hao ZQ, Hargrove WW, Hart TB, Hau BCH, He FL, Hoffman FM, Howe RW, Hubbell SP, Inman-Narahari FM, Jansen PA, Jiang MX, Johnson DJ, Kanzaki M, Kassim AR, Kenfack D, Kibet S, Kinnaird MF, Korte L, Kral K, Kumar J, Larson AJ, Li YD, Li XK, Liu SR, Lum SKY, Lutz JA, Ma KP, Maddalena DM, Makana JR, Malhi Y, Marthews T, Mat Serudin R, McMahon SM, McShea WJ, Memiaghe HR, Mi XC, Mizuno T, Morecroft M, Myers JA, Novotny V, de Oliveira AA, Ong PS, Orwig DA, Ostertag R, den Ouden J, Parker GG, Phillips RP, Sack L, Sainge MN, Sang WG, Sri-Ngernyuang K, Sukumar R, Sun IF, Sungpalee W, Suresh HS, Tan S, Thomas SC, Thomas DW, Thompson J, Turner BL, Uriarte M, Valencia R, Vallejo MI, Vicentini A, Vrška T, Wang XH, Wang XG, Weiblen G, Wolf A, Xu H, Yap S, Zimmerman J (2015) CTFS-ForestGEO: A worldwide network monitoring forests in an era of global change. Global Change Biology, 21, 528-549.

Asner GP, Vitousek PM (2005) Remote analysis of biological invasion and biogeochemical change. Proceedings of the National Academy of Sciences, USA, 102, 4383-4386.

Asner GP, Martin RE, Ford AJ, Metcalfe DJ, Liddell MJ (2009) Leaf chemical and spectral diversity in Australian tropical forests. Ecological Applications, 19, 236-253.

Asner GP, Martin RE, Anderson CB, Knapp DE (2015) Quantifying forest canopy traits: Imaging spectroscopy versus field survey. Remote Sensing of Environment, 158, 15-27.

Asner GP, Knapp DE, Anderson CB, Martin RE, Vaughn N (2016) Large-scale climatic and geophysical controls on the leaf economics spectrum. Proceedings of the National Academy of Sciences, USA, 113, E4043-E4051.

Asner GP, Martin RE, Knapp DE, Tupayachi R, Anderson CB, Sinca F, Vaughn NR, Llactayo W (2017) Airborne laser-guided imaging spectroscopy to map forest trait diversity and guide conservation. Science, 355, 385-389.

Ballanti L, Blesius L, Hines E, Kruse B (2016) Tree species classification using hyperspectral imagery: A comparison of two classifiers. Remote Sensing, 8, 445.

Bongalov B, Burslem DFRP, Jucker T, Thompson SED, Rosindell J, Swinfield T, Nilus R, Clewley D, Phillips OL, Coomes DA (2019) Reconciling the contribution of environmental and stochastic structuring of tropical forest diversity through the lens of imaging spectroscopy. Ecology Letters, 22, 1608-1619.

Cayuela L, Benayas JMR, Justel A, Salas-Rey J (2006) Modelling tree diversity in a highly fragmented tropical montane landscape. Global Ecology and Biogeography, 15, 602-613.

Chen YS, Jiang HL, Li CY, Jia XP, Ghamisi P (2016) Deep feature extraction and classification of hyperspectral images based on convolutional neural networks. IEEE Transactions on Geoscience and Remote Sensing, 54, 6232-6251.

Chesson P, Donahue MJ, Melbourne B, Sears ALW (2005) Scale Transition Theory for Understanding Mechanisms in Metacommunities. University of Chicago Press, Chicago.

Christin S, Hervet É, Lecomte N (2019) Applications for deep learning in ecology. Methods in Ecology and Evolution, 10, 1632-1644.

Christin S, Hervet É, Lecomte N (2021) Going further with model verification and deep learning. Methods in Ecology and Evolution, 12, 130-134.

Clark ML, Roberts DA (2012) Species-level differences in hyperspectral metrics among tropical rainforest trees as determined by a tree-based classifier. Remote Sensing, 4, 1820-1855.

Crawley MJ (2012) The R Book, 2nd edn. Wiley, New Jersey.

Dalponte M, Ørka HO, Ene LT, Gobakken T, Næsset E (2014) Tree crown delineation and tree species classification in boreal forests using hyperspectral and ALS data. Remote Sensing of Environment, 140, 306-317.

Dalponte M, Ene LT, Marconcini M, Gobakken T, Næsset E 
(2015) Semi-supervised SVM for individual tree crown species classification. ISPRS Journal of Photogrammetry and Remote Sensing, 110, 77-87.

Dalponte M, Frizzera L, Gianelle D (2019) Individual tree crown delineation and tree species classification with hyperspectral and LiDAR data. PeerJ, 6, e6227.

Du PJ, Xia JS, Xue ZH, Tan K, Su HJ, Bao R (2016) Review of hyperspectral remote sensing image classification. Journal of Remote Sensing, 20, 236-256. (in Chinese with English abstract) [杜培军, 夏俊士, 薛朝辉, 谭琨, 苏红军, 鲍芯 (2016) 高光谱遥感影像分类研究进展. 遥感学报, 20, 236-256.]

Duro DC, Coops NC, Wulder MA, Han T (2007) Development of a large area biodiversity monitoring system driven by remote sensing. Progress in Physical Geography: Earth and Environment, 31, 235-260.

Fairbanks DHK, McGwire KC (2004) Patterns of floristic richness in vegetation communities of California: Regional scale analysis with multi-temporal NDVI. Global Ecology and Biogeography, 13, 221-235.

Fricker GA, Ventura JD, Wolf JA, North MP, Davis FW, Franklin J (2019) A convolutional neural network classifier identifies tree species in mixed-conifer forest from hyperspectral imagery. Remote Sensing, 11, 2326.

Ghamisi P, Maggiori E, Li ST, Souza R, Tarablaka Y, Moser G, De Giorgi A, Fang LY, Chen YS, Chi MM, Serpico SB, Benediktsson JA (2018) New frontiers in spectral-spatial hyperspectral image classification: The latest advances based on mathematical morphology, Markov random fields, segmentation, sparse representation, and deep learning. IEEE Geoscience and Remote Sensing Magazine, 6(3), 10-43.

Ghosh A, Fassnacht FE, Joshi PK, Koch B (2014) A framework for mapping tree species combining hyperspectral and LiDAR data: Role of selected classifiers and sensor across three spatial scales. International Journal of Applied Earth Observation and Geoinformation, 26, 49-63.

Graves SJ, Asner GP, Martin RE, Anderson CB, Colgan MS, Kalantari L, Bohlman SA (2016) Tree species abundance predictions in a tropical agricultural landscape with a supervised classification model and imbalanced data. Remote Sensing, 8, 161.

Guo QH, Wu FF, Hu TY, Chen LH, Liu J, Zhao XQ, Gao S, Pang SJ (2016) Perspectives and prospects of unmanned aerial vehicle in remote sensing monitoring of biodiversity. Biodiversity Science, 24, 1267-1278. (in Chinese with English abstract) [郭庆华, 吴芳芳, 胡天宇, 陈琳海, 刘 瑾, 赵晓倩, 高上, 庞树金金 (2016) 无人机在生物多样性 遥感监测中的应用现状与展望. 生物多样性, 24, 1267-1278.]

Hu JB, Zhang J (2018) Unmanned aerial vehicle remote sensing in ecology: Advances and prospects. Acta Ecologica
Sinica, 38, 20-30. (in Chinese with English abstract) [胡健 波, 张健 (2018) 无人机遥感在生态学中的应用进展. 生 态学报, 38, 20-30.]

Hu W, Huang YY, Wei L, Zhang F, Li HC (2015) Deep convolutional neural networks for hyperspectral image classification. Journal of Sensors, 2015(2), 1-12.

Huang Y, Chen XH, Liu YL, Sun M, Su QC, Li YD (2019) UAV hyperspectral built-in push-scan image fast splicing method. Journal of Geomatics, 44(5), 24-28. (in Chinese with English abstract) [黄宇, 陈兴海, 刘业林, 孙梅, 苏秋 城, 李艳大 (2019) 基于无人机高光谱影像的引黄灌区 水稻叶片全氮含量估测. 测绘地理信息, 44(5), 24-28.]

Jia W, Pang Y, Ju HB, Li ZY (2018) A BRDF normalization correction method for airborne push broom hyperspectral images in forest areas: China, CN201711429219.3. (in Chinese) [荚文, 庞勇, 鞠洪波, 李增元 (2018) 林区机载 推扫式高光谱影像的BRDF归一化校正方法: 中国, CN201711429219.3.]

Kalacska M, Sanchez-Azofeifa GA, Rivard B, Caelli T, White HP, Calvo-Alvarado JC (2007) Ecological fingerprinting of ecosystem succession: Estimating secondary tropical dry forest structure and diversity using imaging spectroscopy. Remote Sensing of Environment, 108, 82-96.

Kayet N, Pathak K, Chakrabarty A, Kumar S, Singh CP, Chowdary VM (2020) Assessment of mining activities on tree species and diversity in hilltop mining areas using Hyperion and Landsat data. Environmental Science and Pollution Research, 27, 42750-42766.

Kerr JT, Ostrovsky M (2003) From space to species: Ecological applications for remote sensing. Trends in Ecology and Evolution, 18, 299-305.

Kong JX, Zhang ZC, Zhang J (2019) Classification and identification of plant species based on multi-source remote sensing data: Research progress and prospect. Biodiversity Science, 27, 796-812. (in Chinese with English abstract) [孔 嘉金鍂, 张昭臣, 张健 (2019) 基于多源遥感数据的植物物 种分类与识别: 研究进展与展望. 生物多样性, 27 , 796-812.]

Luoto M, Virkkala R, Heikkinen RK, Rainio K (2004) Predicting bird species richness using remote sensing in boreal agricultural-forest mosaics. Ecological Applications, 14, 1946-1962.

Nagendra H (2001) Using remote sensing to assess biodiversity. International Journal of Remote Sensing, 22, 2377-2400.

Oindo BO, Skidmore AK (2002) Interannual variability of NDVI and species richness in Kenya. International Journal of Remote Sensing, 23, 285-298.

$\mathrm{Pu}$ RL (2009) Broadleaf species recognition with in situ hyperspectral data. International Journal of Remote Sensing, 30, 2759-2779.

Qin ZF, Chang QR, Xie BN, Shen J (2016) Rice leaf nitrogen content estimation based on hysperspectral imagery of UAV 
in Yellow River diversion irrigation district. Transactions of the Chinese Society of Agricultural Engineering, 32(23), 77-85. (in Chinese with English abstract) [秦占飞, 常庆瑞, 谢宝妮, 申健 (2016) 基于无人机高光谱影像的引黄灌 区水稻叶片全氮含量估测. 农业工程学报, 32(23), 77-85.]

Quan XW (2017) Research on weak sensitive parameters retrieval using vegetation canopy reflectance model. $\mathrm{PhD}$ dissertation, University of Electronic Science and Technology of China, Chengdu. (in Chinese with English abstract) [全兴文 (2017) 植被冠层反射率模型弱敏感参 数遥感反演方法. 博士学位论文, 电子科技大学, 成都.]

Raczko E, Zagajewski B (2018) Tree species classification of the UNESCO Man and the Biosphere Karkonoski National Park (Poland) using artificial neural networks and APEX hyperspectral images. Remote Sensing, 10, 1111.

Rivard B, Sanchez-Azofeifa GA, Foley S, Calvo-Alvarado J (2008) Species classification of tropical tree leaf reflectance and dependence on selection of spectral bands. In: Hyperspectral Remote Sensing of Tropical and Sub-tropical Forests (eds Kalacska M, Sanchez-Azofeifa GA), pp. 141-157. CRC Press, Florida.

Shen X, Cao L (2017) Tree-species classification in subtropical forests using airborne hyperspectral and LiDAR data. Remote Sensing, 9, 1180.

Signoroni A, Savardi M, Baronio A, Benini S (2019) Deep learning meets hyperspectral image analysis: A multidisciplinary review. Journal of Imaging, 5, 52.

Song YC (2001) Vegetation Ecology. East China Normal University Press, Shanghai. (in Chinese) [宋永昌 (2001) 植被生态学. 华东师范大学出版社, 上海.]

Tan BX, Li ZY, Chen EX, Pang Y, Wu HG (2008) Research advance in forest information extraction from hyperspectral remote sensing data. Forest Research, 21(A1), 105-111. (in Chinese with English abstract) [谭炳香, 李增元, 陈尔学, 庞勇, 武红敢 (2008) 高光谱遥感森林信息提取研究进 展. 林业科学研究, 21(A1), 105-111.]

Tang ZY, Jiang MW, Zhang J, Zhang XY (2018) Applications of satellite and air-borne remote sensing in biodiversity research and conservation. Biodiversity Science, 26, 807-818. (in Chinese with English abstract) [唐志尧, 蒋旻 炜, 张健, 张新悦 (2018) 航空航天遥感在物种多样性研 究与保护中的应用. 生物多样性, 26, 807-818.]

Thenkabail PS, Lyon JG, Huete A (2018) Biophysical and Biochemical Characterization and Plant Species Studies, 2nd edn. CRC Press, Taylor \& Francis Group, Florida.

Trier ØD, Salberg AB, Kermit M, Rudjord Ø, Gobakken T, Næsset E, Aarsten D (2018) Tree species classification in Norway from airborne hyperspectral and airborne laser scanning data. European Journal of Remote Sensing, 51, 336-351.

Turner W, Spector S, Gardiner N, Fladeland M, Sterling E, Steininger M (2003) Remote sensing for biodiversity science and conservation. Trends in Ecology and Evolution, 18, 306-314.

Underwood E, Ustin S, DiPietro D (2003) Mapping nonnative plants using hyperspectral imagery. Remote Sensing of Environment, 86, 150-161.

Vaglio Laurin G, Liesenberg V, Chen Q, Guerriero L, Del Frate F, Bartolini A, Coomes D, Wilebore B, Lindsell J, Valentini R (2013) Optical and SAR sensor synergies for forest and land cover mapping in a tropical site in West Africa. International Journal of Applied Earth Observation and Geoinformation, 21, 7-16.

Wäldchen J, Mäder P (2018) Machine learning for image based species identification. Methods in Ecology and Evolution, 9, 2216-2225.

Wang BC, Smith TB (2002) Closing the seed dispersal loop. Trends in Ecology and Evolution, 17, 379-386.

Wu JG (2007) Landscape Ecology: Pattern, Process, Scale and Hierarchy, 2nd edn. Higher Education Press, Beijing. (in Chinese) [邬建国 (2007) 景观生态学一格局、过程、尺 度与等级 (第二版). 高等教育出版社, 北京.]

Xi XF, Zhou GD (2016) A survey on deep learning for natural language processing. Acta Automatica Sinica, 42, 1445-1465. (in Chinese with English abstract) [奚雪峰, 周 国栋 (2016) 面向自然语言处理的深度学习研究. 自动 化学报, 42, 1445-1465.]

Xi YB, Ren CY, Wang ZM, Wei SQ, Bai JL, Zhang B, Xiang HX, Chen L (2019) Mapping tree species composition using OHS-1 hyperspectral data and deep learning algorithms in Changbai Mountains, Northeast China. Forests, 10, 818.

Yang C, Wu GF, Li QQ, Wang JL, Qu LQ, Ding K (2018) Research progress on remote sensing classification of vegetation. Geography and Geo-Information Science, 34(4), 24-32. (in Chinese with English abstract) [杨超, 鸟国锋, 李清泉, 王金亮, 渠立权, 丁凯 (2018) 植被遥感分类方 法研究进展. 地理与地理信息科学, 34(4), 24-32.

Zhang CY, Qiu F (2012) Mapping individual tree species in an urban forest using airborne lidar data and hyperspectral imagery. Photogrammetric Engineering \& Remote Sensing, 78, 1079-1087.

Zhang J, Chen SB, Chen B, Du YJ, Huang XL, Pan XB, Zhang Q (2013) Citizen science: Integrating scientific research, ecological conservation and public participation. Biodiversity Science, 21, 738-749. (in Chinese with English abstract) [张健, 陈圣宾, 陈涁, 杜彦君, 黄晓䂞, 潘绪斌, 张强 (2013) 公众科学: 整合科学研究、生态保护和公众 参与. 生物多样性, 21, 738-749.]

Zhang YJ, Fan CK, Huang K, Liu YJ, Zu JX, Zhu JT (2017) Opportunities and challenges in remote sensing applications to ecosystem ecology. Chinese Journal of Ecology, 36, 809-823. (in Chinese with English abstract) [张扬建, 范春 捆, 黄珂, 刘瑶杰, 组佳星, 朱军涛 (2017) 遥感在生态 系统生态学上应用的机遇与挑战. 生态学杂志, 36, 809-823.] 
Zhang ZM, Xu Q, Wang B, Sun H, Geng YP, Tian J (2017) Applications of unmanned aerial vehicles remote sensing technology in landscape ecology. Acta Ecologica Sinica, 37, 4029-4036. (in Chinese with English abstract) [张志明, 徐 倩, 王彬, 孙虎, 耿宇鹏, 田冀 (2017) 无人机遥感技术 在景观生态学中的应用. 生态学报, 37, 4029-4036.]

Zhao SD, Zhang YM (2006) Ecosystems and human well-being: The achievements, contributions and prospects of the millennium ecosystem assessment. Advances in Earth Science, 21, 895-902. (in Chinese with English abstract) [赵 士洞，张永民 (2006) 生态系统与人类福祉——千年生 态系统评估的成就、贡献和展望. 地球科学进展, 21, 895-902.]

\section{附录 Supplementary Material}

附录1 高光谱无人机近地面遥感系统

Appendix 1 Hyperspectral UAV near ground remote sensing system https://www.biodiversity-science.net/fileup/PDF/2021013-1.pdf

\section{附录2 常用的一站式行业航测图像分析软件}

Appendix 2 Commonly used one-stop industry aerial survey image analysis software https://www.biodiversity-science.net/fileup/PDF/2021013-1.pdf 


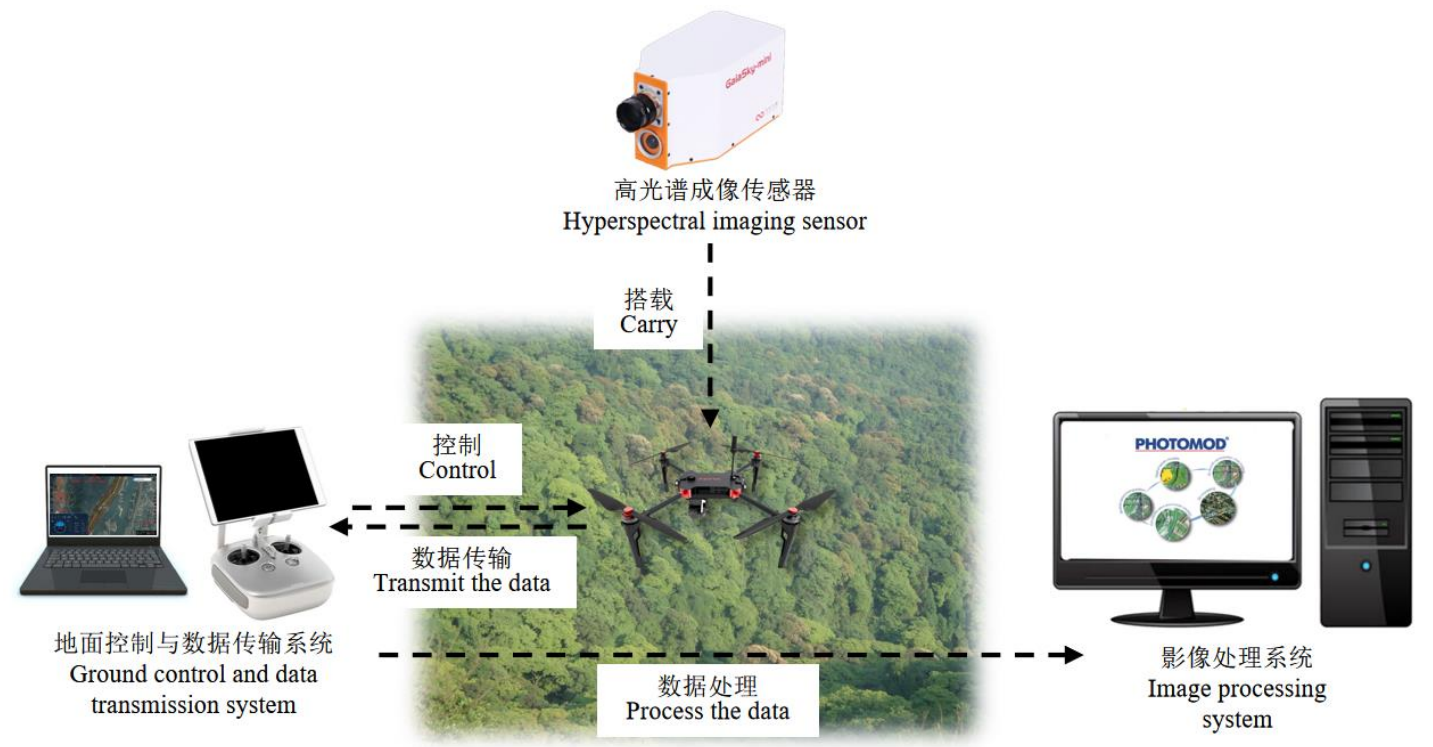

附录1 高光谱无人机近地面遥感系统。在这一系统中, 无人机低空飞行平台可搭载高光谱成像传感器, 通 过地面控制与数据传输系统监测无人机的飞行状态和传感器的信息。高光谱成像系统采集的信息可以通过 影像处理系统进行拼接处理, 生成各类图像和模型。

Appendix 1 Hyperspectral UAV near ground remote sensing system. In this system, the UAV low altitude flight platform can be equipped with hyperspectral imaging sensors to monitor the flight status of UAV and sensor information through the ground control and data transmission system. The information collected by hyperspectral imaging system can be spliced by image processing system to generate various images and models. 
徐岩, 张聪伶, 降瑞娇, 王子斐, 朱梦晨, 沈国春 (2021) 无人机高光谱影像与冠层树种多样性监测. 生物 多样性, 29, 647-660. http://www.biodiversity-science.net/CN/10.17520/biods.2021013

附录2 常用的一站式行业航测图像分析软件

Appendix 2 Commonly used one-stop industry aerial survey image analysis software

\begin{tabular}{|c|c|}
\hline 软件 Software & 介绍 Introduction \\
\hline Pix4D & $\begin{array}{l}\text { Pix4D软件是目前应用较为广泛的无人机摄影测量软件, 具备高精度、高效率、全自动工作流程和高 } \\
\text { 质量的成果, 可以实现从无人机图像采集到数据处理再到数据分析整个专业的工作流程, 生成高精 } \\
\text { 度并带地理坐标的二维地图和三维模型, 如数字地表模型、正射影像镶嵌图、三维纹理模型等。 }\end{array}$ \\
\hline PhotoScan & $\begin{array}{l}\text { PhotoScan是一款基于影像自动生成高质量模型的软件, 可生成高分辨率真正射影像及带精细色彩纹 } \\
\text { 理的DEM模型。通过完全自动化的工作流程, 即使非专业人员也可以在一台电脑上处理成百上千张 } \\
\text { 航空影像, 生成专业级别的摄影测量数据。 }\end{array}$ \\
\hline 飞马无人机管家 & $\begin{array}{l}\text { 飞马无人机管家是一站式智能GIS系统, 拥有三维航线智能规划、飞行状态实时三维呈现、无人机数 } \\
\text { 据智能预处理、智能高精度快速全成果处理等功能。能够输出高精度、高质量的DSM、DOM及实 } \\
\text { 三维模型, 并可通过激光点云数据处理输出DEM、等高线等成果。 }\end{array}$ \\
\hline 大疆智图 & $\begin{array}{l}\text { 大疆智图是一款提供自主航线规划、飞行航拍、二维正射影像与三维模型重建的PC应用软件。一站 } \\
\text { 式解决方案帮助行业用户全面提升航测内外业效率, 将真实场景转化为数字资产。 }\end{array}$ \\
\hline ENVI OneButton & $\begin{array}{l}\text { ENVI OneButton可通过全自动方式来处理无人机拍摄的图像, 利用先进的摄影测量和计算机视觉算 } \\
\text { 法, 采用空三加密和区域网平差技术快速得到高精度、具备标准地理参考、无缝镶嵌的正射影像、地 } \\
\text { 形和真彩色3D点云产品。 }\end{array}$ \\
\hline LiMapper & $\begin{array}{l}\text { LiMapper是一款航空摄影测量软件, 具有数据自动化处理、高效稳定运行、成果精度高等特点, 拥有 } \\
\text { 特征点提取匹配、区域网平差、相机自检校、密集点云重建、DEM/DSM生成、正射影像智能镶嵌、 } \\
\text { 真正射影像生成、拼接线编辑以及数据可视化分析等功能, 并采用并行架构和GPU高性能运算, 能够 } \\
\text { 基于重叠的影像数据恢复物体精细的三维几何结构, 生成一系列标准的测绘成果。 }\end{array}$ \\
\hline Inpho & $\begin{array}{l}\text { Inpho软件采用先进独特的摄影测量技术, 把原始的航拍图像和卫星图像精确地转换成连贯准确的 } \\
\text { 点云和地表模型、正射影像和三维的特征地物。Inpho软件采取模块化结构, 既可以整合成完整的工 } \\
\text { 作流程, 也可以采用其中一些独立的组件整合到空间地理信息的生产工作流中。 }\end{array}$ \\
\hline
\end{tabular}

